Received Date : 14-Apr-2015

Accepted Date : 20-Apr-2015

Article type : Editorial

Corresponding author mail id: andrea.banfi@usb.ch

\title{
VEGF, shear stress and muscle angiogenesis: a complicated triangle
}

\author{
Andrea Banfi ${ }^{1}$ and Roberto Gianni-Barrera ${ }^{1}$ \\ ${ }^{1}$ Cell and Gene Therapy, Department of Biomedicine, University of Basel, and Department \\ of Surgery, Basel University Hospital, Basel, Switzerland
}

In this issue of Acta Physiologica, Uchida and colleagues (Uchida et al., 2015) report interesting findings that help clarify the complex relationship between flow-induced shear stress, expression of Vascular Endothelial Growth Factor-A (VEGF) and angiogenesis in skeletal muscle; a relationship that has been particularly elusive to elucidate due to the multiple and mutual influences among these factors.

Stimulation of muscle angiogenesis is a therapeutic goal to restore blood flow to the ischemic limbs of patients suffering from peripheral artery disease (PAD). While the delivery of VEGF and other angiogenic factors to the affected muscles is an active area of on-going research, no therapeutic angiogenesis approach has yet entered clinical practice. On the other hand, exercise training has been shown to very effectively increase vessel density in trained muscles, through stimulation of VEGF expression. Actually, exercise training is currently the only clinically effective non-surgical therapy for PAD and the most recent consensus guidelines of the American College of Cardiology Foundation and the American Heart Association recommend exercise training as the first line of treatment for patients with symptomatic PAD and intermittent claudication This article has been accepted for publication and undergone full peer review but has not been through the copyediting, typesetting, pagination and proofreading process which may lead to differences between this version and the Version of Record. Please cite this article as an 'Accepted Article', doi: 10.1111/apha.12514 This article is protected by copyright. All rights reserved. 
(Rooke et al., 2013). The effects of exercise on muscle angiogenesis are complex and involve both metabolic (reduced tissue levels of oxygen and nutrients) and hemodynamic signals (vasodilation with increased blood flow and shear stress). However, while both factors increase VEGF expression, they have been described to cause vessel growth by very different processes, namely sprouting angiogenesis and vascular splitting or intussusception, respectively.

Sprouting relies on the specification of endothelial cells into tip and stalk. Tip cells do not have a lumen, extend numerous thin filopodia, through which they can sense VEGF gradients, and migrate towards the VEGF source, thereby guiding the nascent sprout. Tip cells activate Notch signalling in the neighbouring endothelial cells, causing them to become instead stalk cells, which proliferate just behind the tip, form the body of the sprout and start the process of lumen formation (Blanco and Gerhardt, 2013). On the other hand, vascular splitting and intussusception represent the conceptual opposite of sprouting, as endothelial cells extend inside the lumen to split it into two new daughter vessels. Hallmark of these processes is the formation of trans-luminal tissue pillars, which can occur either through a zone of contact between the endothelial cells of opposite capillary walls, with subsequent reorganization of the endothelial junctions and invasion of the pillar core by myofibroblasts (intussusception), or through the extension and fusion of intraluminal protrusions made exclusively of endothelial cells (splitting). Subsequently, transluminal tissue pillars align along the length of the preexisting vessel, progressively fuse together and divide the affected vascular segment longitudinally (Egginton, 2009, Makanya et al., 2009). It is not completely clear, and the subject of on-going discussion, whether intussusception and vascular splitting are two distinct mechanisms or different aspects of the same general process. For simplicity, here we will use the term intussusception to mean both.

The Authors investigated how shear stress induces angiogenesis in muscle in the absence of metabolic changes, using a classic model based on systemic treatment with the $\alpha 1$-adrenergic receptor blocker prazosin, which increases blood flow and shear stress in the downstream microvascular networks purely through arterial vasodilation. The widely held view has been that, while metabolic stimuli cause VEGF expression from muscle fibers, intussusception induced by hemodynamic factors depends on VEGF

This article is protected by copyright. All rights reserved. 
produced by the endothelial cells themselves, which are the only cell type exposed to shear stress and can sense it directly. However, the Authors' findings shed new light on this dogma: by taking advantage of a knock-out mouse model lacking VEGF specifically in skeletal myocytes, but not other cell types, they elegantly showed that the angiogenesis caused by a chronic increase in blood flow and shear stress in skeletal muscles unexpectedly requires myocyte-derived VEGF and endothelial VEGF cannot compensate for its absence (Uchida et al., 2015). Further, they found that Nitric Oxide (NO), which is known to be released by endothelium in response to augmented shear stress (Hudlicka and Brown, 2009), could stimulate VEGF expression independently of flow, in ex vivo isolated muscles, as well as in cultured myocytes. These findings are highly suggestive for a potential mechanism whereby shear stress, sensed by endothelium, may stimulate VEGF expression by the adjacent muscle fibres through NO diffusion in the microenvironment of the vessels: this interesting concept certainly deserves further study.

VEGF produced by myofibers, i.e. outside of the blood vessels in skeletal muscle, has been thought to be important to direct sprouting angiogenesis, which requires a VEGF gradient to guide vascular growth, whereas intussusceptive angiogenesis, which is wholly contained within the vessel itself, has been believed to rely on endothelialderived VEGF induced by shear stress. Now, the findings by Uchida et al. show that myofiber-derived VEGF is the key inducer also for shear-dependent intussusceptive angiogenesis. However, the contradiction may be only apparent. In fact, it should be considered that muscle is a highly compact tissue, whose closely arrayed structure of parallel myofibers poses significant spatial constraints to the migration of sprouting tips (reviewed in (Haas et al., 2012)), and it is unclear whether VEGF gradients may actually form within the very limited amounts of matrix present between fibers and capillaries. Landmark experiments from over a decade ago elegantly showed how spatial distribution of VEGF in the matrix controls vascular morphogenesis (Ruhrberg et al., 2002) and computational models suggest that saturation of the matrix around myofibers with VEGF, without the formation of any gradient, would lead to circumferential enlargement rather than sprouting (Bentley et al., 2008, Gianni-Barrera et al., 2011). Indeed, recent evidence shows that VEGF over-expression by skeletal muscle fibers, at the doses required to induce functional benefit and restore tissue perfusion after

This article is protected by copyright. All rights reserved. 
ischemia, induces angiogenesis essentially without sprouting and rather through vascular enlargement followed by intussusception (Gianni-Barrera et al., 2013).

The findings by Uchida et al. contribute new understanding on the link between shear stress, VEGF and intussusception and, based on these results, VEGF expression from the muscle fibers, leading to circumferential enlargement of vessels followed by longitudinal splitting rather than sprouting, may be a common mechanism for therapeutic angiogenesis induced both by exercise and shear stress on one hand, and by VEGF gene delivery on the other. Unfortunately, the molecular mechanisms of VEGF-induced intussusceptive angiogenesis remain poorly understood, compared to those regulating sprouting, mainly due to a paucity of appropriate models. Studies like this, exploiting well-controlled models and defined genetic manipulations, will hopefully contribute to bridge this gap and identify rational molecular targets for therapeutic angiogenesis.

\section{Conflict of Interest}

No conflict of interest to declare.

\section{Acknowledgement}

This work was supported by a grant of the Swiss National Science Foundation (310030_143898) to A.B. and a grant by the Swiss Heart Foundation to R.G.-B.

\section{References}

Bentley, K., Gerhardt, H. \& Bates, P. A. 2008. Agent-based simulation of notch-mediated tip cell selection in angiogenic sprout initialisation. Journal of theoretical biology, 250, 25-36.

Blanco, R. \& Gerhardt, H. 2013. VEGF and Notch in tip and stalk cell selection. Cold Spring Harbor perspectives in medicine, 3, a006569.

Egginton, S. 2009. Invited review: activity-induced angiogenesis. Pflugers Archiv : European journal of physiology, 457, 963-77.

Gianni-Barrera, R., Trani, M., Fontanellaz, C., Heberer, M., Djonov, V., Hlushchuk, R. \& Banfi, A. 2013. VEGF over-expression in skeletal muscle induces angiogenesis by intussusception rather than sprouting. Angiogenesis, 16, 123-36.

Gianni-Barrera, R., Trani, M., Reginato, S. \& Banfi, A. 2011. To sprout or to split? VEGF, Notch and vascular morphogenesis. Biochemical Society transactions, 39, 1644-8.

Haas, T. L., Lloyd, P. G., Yang, H. T. \& Terjung, R. L. 2012. Exercise training and peripheral arterial disease. Comprehensive Physiology, 2, 2933-3017.

Hudlicka, 0. \& Brown, M. D. 2009. Adaptation of skeletal muscle microvasculature to increased or decreased blood flow: role of shear stress, nitric oxide and vascular endothelial growth factor. Journal of vascular research, 46, 504-12.

This article is protected by copyright. All rights reserved. 
Makanya, A. N., Hlushchuk, R. \& Djonov, V. G. 2009. Intussusceptive angiogenesis and its role in vascular morphogenesis, patterning, and remodeling. Angiogenesis, 12, 113-23.

Rooke, T. W., Hirsch, A. T., Misra, S., Sidawy, A. N., Beckman, J. A., Findeiss, L., Golzarian, J., Gornik, H. L., Jaff, M. R., Moneta, G. L., Olin, J. W., Stanley, J. C., White, C. J., White, J. V. \& Zierler, R. E. 2013. Management of patients with peripheral artery disease (compilation of 2005 and 2011 ACCF/AHA Guideline Recommendations): a report of the American College of Cardiology Foundation/American Heart Association Task Force on Practice Guidelines. Journal of the American College of Cardiology, 61, 1555-70.

Ruhrberg, C., Gerhardt, H., Golding, M., Watson, R., Ioannidou, S., Fujisawa, H., Betsholtz, C. \& Shima, D. T. 2002. Spatially restricted patterning cues provided by heparinbinding VEGF-A control blood vessel branching morphogenesis. Genes \& development, 16, 2684-98.

Uchida, C., Nwadozi, E., Hasanee, A., Olenich, S., Olfert, I. M. \& Haas, T. L. 2015. Musclederived vascular endothelial growth factor regulates microvascular remodelling in response to increased shear stress in mice. Acta physiologica.

This article is protected by copyright. All rights reserved. 